\title{
Accuracy of the Simplified Nutritional Appetite Questionnaire for Malnutrition and Sarcopenia Screening among Older Patients Requiring Rehabilitation
}

\author{
Akio Shimizu ${ }^{1,2,3} \mathbb{C}^{\mathbb{D}}$, Ichiro Fujishima ${ }^{4}$, Keisuke Maeda ${ }^{2,3, * \mathbb{D}}$, Kenta Murotani ${ }^{5} \mathbb{D}$, Tatsuro Inoue ${ }^{3,6} \mathbb{D}$, \\ Tomohisa Ohno ${ }^{7} \mathbb{D}$, Akiko Nomoto ${ }^{7}$, Junko Ueshima ${ }^{2,8} \mathbb{D}^{\mathbb{D}}$, Yuria Ishida ${ }^{2,9}$, Ayano Nagano ${ }^{2,10} \mathbb{D}$, Jun Kayashita 11 \\ and Naoharu Mori ${ }^{2}$ (D)
}

Citation: Shimizu, A.; Fujishima, I.; Maeda, K.; Murotani, K.; Inoue, T.; Ohno, T.; Nomoto, A.; Ueshima, J.; Ishida, Y.; Nagano, A.; et al. Accuracy of the Simplified Nutritional Appetite Questionnaire for Malnutrition and Sarcopenia Screening among Older Patients Requiring Rehabilitation. Nutrients 2021, 13, 2738. https:// doi.org/10.3390/nu13082738

Academic Editors: Giuseppe Sergi and Andrea P. Rossi

Received: 23 June 2021

Accepted: 5 August 2021

Published: 10 August 2021

Publisher's Note: MDPI stays neutra with regard to jurisdictional claims in published maps and institutional affiliations.

Copyright: (C) 2021 by the authors Licensee MDPI, Basel, Switzerland. This article is an open access article distributed under the terms and conditions of the Creative Commons Attribution (CC BY) license (https:/ / creativecommons.org/licenses/by/ $4.0 /)$
1 Department of Nutrition, Hamamatsu City Rehabilitation Hospital, Hamamatsu 433-8127, Japan; a.shimizu.diet@gmail.com

2 Department of Palliative and Supportive Medicine, Graduate School of Medicine, Aichi Medical University, Nagakute 480-1195, Japan; j.ueshima@gmail.com (J.U.); okuda.yuria.785@mail.aichi-med-u.ac.jp (Y.I.); aya.k.nagano@gmail.com (A.N.); nmori@aichi-med-u.ac.jp (N.M.)

3 Department of Geriatric Medicine, Hospital, National Center for Geriatrics and Gerontology, Obu 474-8511, Japan; tatsuro-inoue@nuhw.ac.jp

4 Department of Rehabilitation Medicine, Hamamatsu City Rehabilitation Hospital, Hamamatsu 433-8127, Japan; ifujishima@sis.seirei.or.jp

5 Biostatistics Center, Kurume University, Kurume 830-0011, Japan; kmurotani@med.kurume-u.ac.jp

6 Department of Physical Therapy, Niigata University of Health and Welfare, Niigata 950-3198, Japan

7 Department of Dentistry, Hamamatsu City Rehabilitation Hospital, Hamamatsu 433-8127, Japan; tomohisa@zd5.so-net.ne.jp (T.O.);n.akiko-dent@sis.seirei.or.jp (A.N.)

8 Department of Clinical Nutrition and Food Service, NTT Medical Center Tokyo, Tokyo 141-8625, Japan

9 Department of Nutrition, Aichi Medical University, Nagakute 480-1195, Japan

10 Department of Nursing, Nishinomiya Kyoritsu Neurosurgical Hospital, Nishinomiya 663-8211, Japan

11 Department of Health Sciences, Faculty of Human Culture and Science, Prefectural University of Hiroshima, Hiroshima 734-8558, Japan; kayashita@pu-hiroshima.ac.jp

* Correspondence: kskmaeda1701@gmail.com; Tel.: +81-561-62-3311; Fax: +81-561-78-6364

\begin{abstract}
This cross-sectional study aimed to examine the accuracy of the Simplified Nutritional Appetite Questionnaire (SNAQ) and the SNAQ for Japanese Elderly (SNAQ-JE) for the Global Leadership Initiative on Malnutrition (GLIM)-defined malnutrition and sarcopenia screening in older persons. We included 380 inpatients aged $\geq 65$ years (mean age, $79.3 \pm 7.9 ; 60.0 \%$ women) and admitted to rehabilitation units. Undernutrition and sarcopenia were diagnosed based on GLIM criteria and the Asian Working Group for Sarcopenia, respectively, using bioimpedance analysis. Poor appetite was defined as an SNAQ score of $<14$ points and an SNAQ-JE score of $\leq 14$ points. The sensitivity, specificity, and accuracy of these tools for detecting poor appetite for GLIM-defined malnutrition and sarcopenia were assessed. The rates of GLIM-defined malnutrition and sarcopenia were $56.8 \%$ and $59.2 \%$, respectively. The number of patients with poor appetite was $94(24.7 \%)$ for the SNAQ and $234(61.6 \%)$ for the SNAQ-JE. The sensitivity and specificity of the SNAQ measured against GLIM-defined malnutrition were $32.9 \%$ and $73.1 \%$, respectively, and against sarcopenia were $29.8 \%$ and $70.2 \%$, respectively. The sensitivity and specificity of the SNAQ-JE measured against GLIM-defined malnutrition were $82.6 \%$ and $51.0 \%$, respectively, and against sarcopenia were $86.0 \%$ and $53.7 \%$, respectively. The SNAQ-JE showed fair accuracy for GLIM-defined malnutrition and sarcopenia in older patients admitted to rehabilitation units.
\end{abstract}

Keywords: older adults; sarcopenia; malnutrition; poor appetite

\section{Introduction}

Poor appetite in older adults is recognized as a part of geriatric syndromes [1]. Its causes are multifactorial and include age-related physiological changes, morbidity, and 
social factors [2]. It is associated with physical disability and frailty [3,4] and is thought to lead to adverse outcomes such as morbidity and mortality [5]. In particular, poor appetite may lead to inadequate dietary intake and is associated with undernutrition and sarcopenia [4,6,7]. Early diagnosis and treatment of poor appetite in older people may prevent undernutrition, sarcopenia, frailty, and adverse outcomes.

Many screening tools have been developed for the early detection of undernutrition and sarcopenia. The Mini Nutritional Assessment Tool Short Form (MNA-SF) [8] is a screening tool for undernutrition in older adults that is associated with satisfactory accuracy [9]. Sarcopenia screening tools include SARC-F and calf circumference $[6,10,11]$. These screening tools do not directly assess poor appetite, although they may assess food intake, physical function, and muscle mass [1]. Since poor appetite is associated with undernutrition and sarcopenia, [3,4] screening for poor appetite may improve undernutrition and sarcopenia treatment.

The Simplified Nutritional Appetite Questionnaire (SNAQ) was developed to identify poor appetite in older people [12]. In addition, the SNAQ for Japanese Elderly (SNAQ-JE) has been validated for use in the Japanese older adult population [13]. The SNAQ has been shown to have sensitivity and specificity of approximately $70 \%$ each for predicting undernutrition in older inpatients [14-17]. However, previous studies have not validated the accuracy of the SNAQ against the Global Leadership Initiative on Malnutrition (GLIM) criteria, used to diagnose undernutrition in various regions and ethnicities [7]. The GLIM criteria are highly sensitive in diagnosing undernutrition in older inpatients and have good predictive validity for poor prognosis [18-21]. Therefore, determining the sensitivity and specificity of the SNAQ to the GLIM criteria may provide opportunities for interventions for both poor appetite and undernutrition. Similarly, sarcopenia is associated with worse functional outcomes, such as swallowing function, cognitive function, and activities of daily living (ADLs), in older inpatients [6,22]. Tools that help increase the rate of accurate sarcopenia diagnosis are required in clinical practice.

To the best of our knowledge, no previous study has examined the screening accuracy of the SNAQ and SNAQ-JE for GLIM-defined malnutrition and sarcopenia in older inpatients. Tools to assess poor appetite, including the SNAQ and SNAQ-JE, can predict future weight loss $[12,13]$. Thus, they are expected to play multiple roles, not only for screening current undernutrition and sarcopenia but also for screening patients who may develop or experience worsening undernutrition and sarcopenia in the future. In addition, the SNAQ-JE was developed for better adaptability in the Japanese older adult population [13]. Nevertheless, the SNAQ has been used in some studies of the Japanese older adult population [23]. Hence, we thought it necessary to compare the respective screening accuracies of the SNAQ and SNAQ-JE. Therefore, this study aimed to verify the screening accuracy of the SNAQ and SNAQ-JE for GLIM-defined malnutrition and sarcopenia in older patients.

\section{Materials and Methods}

\subsection{Participants}

This cross-sectional study was performed at the Hamamatsu City Rehabilitation Hospital (Shizuoka, Japan). This hospital provides rehabilitation services based on the Japanese insurance system. The participants were older hospitalized patients with stroke, musculoskeletal disease, or hospital-associated deconditioning, aged $\geq 65$ years, and admitted to our rehabilitation units between January 2019 and January 2021. Patients who were able to answer the appetite questionnaire were included. Patients were excluded if they required non-oral intake, they could not answer the appetite questionnaire, they had pacemaker insertions, their bio-electrical impedance analysis (BIA) could not be measured, or their data collection was incomplete. This study was approved by the Ethics Committee of the Hamamatsu City Rehabilitation Hospital (ID: 20-63). Informed consent was not obtained because of the retrospective study design. Patients could withdraw from the study at any time, using the opt-out notification function available on the study website. 


\subsection{Measurements}

Data on the characteristics of patients were obtained from their medical records, including age, sex, primary diseases, number of days from disease onset to admission in a rehabilitation hospital, height, weight, body mass index (BMI), comorbidities, muscle mass, handgrip strength, swallowing function, cognitive function, and ADLs. The Charlson comorbidity index (CCI) [24], an ordinal scale with weighted scores, was used to evaluate comorbidities, considering diseases that impact prognoses, such as congestive heart failure and malignancy. The higher the score, the worse the prognosis [24]. Height and weight were measured by nurses, and the BMI was calculated by dividing the weight $(\mathrm{kg})$ by the square of the height $\left(\mathrm{m}^{2}\right)$. Muscle mass was measured by a dietitian, using the BIA method as recommended by the Asian Working Group for Sarcopenia (AWGS) 2019 [10]. The BIA method was performed while patients were in the supine position, before lunch, with the bladder emptied and with clothing on. The skeletal muscle mass index was calculated by dividing the appendicular skeletal muscle mass $(\mathrm{kg})$, measured using the BIA method, by the square of the height $\left(\mathrm{m}^{2}\right)$. Handgrip strength was measured using a hand dynamometer (MG-4800; CHARDER Electronic, Taichung, Taiwan). These parameters were measured with the participant sitting in a chair or bed with their elbows bent at $90^{\circ}$. Measurements were taken twice for the right and left hands, and the highest value was used for analysis. Swallowing function was assessed by speech-language therapists, using the Food Intake Level Scale (FILS) [25]. The FILS is an ordinal scale, scored from 1 to 10 points, focusing on daily consumption; higher scores indicate better swallowing function [25]. The ADLs were evaluated by physical or occupational therapists, using the Functional Independence Measure (FIM) [26]. The FIM consists of 18 items, with scores ranging on an ordinal scale from 18 to 126 points; a higher FIM score indicates higher ADLs independence [26]. Cognitive function was assessed by occupational therapists, using the Mini-Mental Examination State (MMSE) [27]. The MMSE score ranged from 0 to 30 points, with lower scores indicating lower cognitive function. This information was obtained within 3 days of admission.

\subsection{Appetite Assessment Tools: SNAQ and SNAQ-JE}

The SNAQ and SNAQ-JE, which have been validated in Japanese older populations, were used to assess appetite $[12,13,28]$. The SNAQ and SNAQ-JE comprise ordinal scales, consisting of four domains: appetite, feeling of fullness, taste of food, and the number of meals per day for the SNAQ [12,28] and appetite, feeling of fullness, taste of food, and "general mood" for the SNAQ-JE [13]. It has been reported that the questionnaire items used in the SNAQ-JE are more widely distributed and have higher factor loadings than those used in the SNAQ for older Japanese populations [13]. The score for each item on the SNAQ and SNAQ-JE ranges from 1 (very poor, very sad, etc.) to 5 (very good, very happy, etc.) $[12,13,28]$. The total score for the SNAQ and SNAQ-JE is in the same range of $4-20$ points $[12,13,28]$. Previous studies have reported that the SNAQ-JE may be more suitable than the SNAQ for use with older Japanese adults [13]. The SNAQ is a simple screening tool with good predictive ability for weight loss [12,29]. A total score of $<14$ points for the SNAQ and $\leq 14$ points for the SNAQ-JE is considered indicative of poor appetite $[12,13,28]$.

\subsection{Diagnosis of GLIM-Defined Malnutrition}

The MNA-SF [8] was used for nutritional screening; this was followed by an assessment of undernutrition using the GLIM diagnostic criteria. The MNA-SF consists of six items and has a score range of $0-14$ points, with scores of $\leq 11$ points indicating a risk of undernutrition [8]. The diagnosis of undernutrition was based on the GLIM criteria [7]. The GLIM criteria consist of phenotypic and etiologic criteria and involve two steps: risk identification and undernutrition diagnosis. In this study, the MNA-SF [8] was used for nutritional screening, and patients with a score of $\leq 11$ points were considered for the diagnostic process. GLIM-defined malnutrition was diagnosed in individuals who met 
at least one of the phenotypic and etiologic criteria. The phenotypic criteria involved weight loss (5-10\% within 6 months or $>10 \%$ beyond 6 months), low BMI $\left(<18.5 \mathrm{~kg} / \mathrm{m}^{2}\right.$ for patients aged $<70$ years or $<20 \mathrm{~kg} / \mathrm{m}^{2}$ for those aged $\geq 70$ years), and decreased muscle mass (skeletal muscle mass index (SMI) of $<7.00 \mathrm{~kg} / \mathrm{m}^{2}$ for males and $<5.70 \mathrm{~kg} / \mathrm{m}^{2}$ for females) [7]. The etiologic criteria involved decreased food intake or assimilation (vomiting and dysphagia) and inflammatory conditions/disease burden (acute or chronic disease) [7].

\subsection{Diagnosis of Sarcopenia}

Patients with decreased muscle mass and low muscle strength were diagnosed with sarcopenia, according to the diagnostic algorithm of the AWGS 2019 [10]. In this study, the walking speed and five times sit-to-stand, which are part of the AWGS 2019 diagnostic criteria, could not be performed in all patients due to their physical disabilities [30]. Therefore, sarcopenia was diagnosed by a combination of low muscle strength and decreased muscle mass. Muscle strength was assessed using handgrip strength, and handgrip strength values of $<28.0 \mathrm{~kg}$ and $<18.0 \mathrm{~kg}$ for men and women, respectively, were defined as low muscle strength. Muscle mass was assessed using SMI; SMI values of $<7.00 \mathrm{~kg} / \mathrm{m}^{2}$ and $<5.70 \mathrm{~kg} / \mathrm{m}^{2}$ for men and women, respectively, were defined as decreased muscle mass.

\subsection{Data Analysis}

Quantitative variables included non-parametric and parametric values and were expressed as median (interquartile range) or mean \pm standard deviation. Categorical variables were expressed as the number of patients (\%). Comparisons between groups were made using the Mann-Whitney $U$ test for non-parametric variables, $t$-test for parametric variables, and $\chi^{2}$ test for categorical variables. Multivariate logistic regression analysis was performed with GLIM-defined malnutrition as the dependent variable in Model 1 and sarcopenia as the dependent variable in Model 2 to determine whether poor appetite assessed by the SNAQ and SNAQ-JE was independently associated. As confounding factors affecting appetite, variables reported in previous studies [31], such as age, sex, primary disease, number of days from disease onset to rehabilitation, comorbidities, cognitive function, swallowing function, nutritional status, and ADLs, were entered. We calculated sensitivity, specificity, accuracy, positive predictive value, and negative predictive value to evaluate the ability of poor appetite to predict the diagnosis of GLIM-defined malnutrition and sarcopenia. Poor appetite was defined as an SNAQ score of $<14$ points and an SNAQ-JE score of $\leq 14$ points, based on previous studies [12,13,28]. In addition, the accuracy for GLIM-defined malnutrition and sarcopenia was analyzed using undernutrition as assessed by an MNA-SF $\leq 7$ as a reference tool. The rating of validity test statistics followed the recommended cut-off points for sensitivity and specificity: a 'good' rating represented a sensitivity and specificity of $>80 \%$; a 'fair' rating represented a sensitivity or specificity of $<80 \%$ but both $>50 \%$; a 'poor' rating represented a sensitivity or specificity of $<50 \%$ [32]. To further assess the ability of the SNAQ and SNAQ-JE to predict the diagnosis of GLIMdefined malnutrition and sarcopenia, the area under the curve (AUC) for the SNAQ and SNAQ-JE was determined, using the receiver operating characteristic curve against GLIMdefined malnutrition and sarcopenia. The AUC values of the SNAQ and SNAQ-JE for GLIM-defined malnutrition and sarcopenia were compared using the DeLong test. All statistical analyses were performed using IBM SPSS version 23.0 (IBM Japan, Tokyo, Japan); $p$-values of $<0.05$ were considered indicative of a statistically significant finding.

\section{Results}

In total, 598 patients were admitted to our rehabilitation units during the study period. Of these, 28 patients had non-oral intake, 163 patients could not answer the appetite questionnaire, 15 patients had missing data, and 12 patients had BIA that could not be measured. Finally, 380 patients (mean age, $79.3 \pm 7.9 ; 60.0 \%$ women) met the eligibility criteria and were included in the analysis. In this study, 94 and 234 participants were classified as having poor appetite in the SNAQ and SNAQ-JE, respectively (Table 1). 
Table 1. Patients' epidemiological characteristics according to appetite status.

\begin{tabular}{|c|c|c|c|c|c|c|}
\hline & SNAQ $<14$ & SNAQ $\geq 14$ & $p$-Value & SNAQ-JE $\leq 14$ & SNAQ-JE > 14 & $p$-Value \\
\hline Number of patients, $n(\%)$ & $94(24.7)$ & $286(75.3)$ & & $234(61.6)$ & $146(38.4)$ & \\
\hline Age, years ${ }^{1}$ & $80.1 \pm 8.1$ & $79.1 \pm 7.8$ & 0.265 & $79.9 \pm 8.0$ & $78.5 \pm 7.7$ & 0.105 \\
\hline Sex (female), $n(\%)^{2}$ & $64(68.1)$ & $164(57.3)$ & 0.070 & $143(61.1)$ & $85(58.2)$ & 0.592 \\
\hline \multicolumn{7}{|l|}{ Primary disease, $n(\%)^{2}$} \\
\hline - Stroke & $36(38.3)$ & $136(47.6)$ & 0.011 & $106(45.3)$ & $66(45.2)$ & 0.365 \\
\hline - Musculoskeletal disease & $47(50.0)$ & $140(49.0)$ & & $112(47.9)$ & $75(51.4)$ & \\
\hline $\begin{array}{l}\text { - Hospital-associated } \\
\text { deconditioning }\end{array}$ & $11(11.7)$ & $10(3.5)$ & & $16(6.8)$ & $5(3.4)$ & \\
\hline $\begin{array}{l}\text { Days from onset to } \\
\text { rehabilitation } \\
3\end{array}$ & $23(15-34)$ & $24(17-34)$ & 0.321 & $24(17-34)$ & $24(17-33)$ & 0.929 \\
\hline $\begin{array}{l}\text { Charlson comorbidity } \\
\text { index, points }{ }^{3}\end{array}$ & $1(0-2)$ & $1(0-2)$ & 0.662 & $1(0-2)$ & $1(0-2)$ & 0.473 \\
\hline Body mass index, $\mathrm{kg} / \mathrm{m}^{21}$ & $20.6 \pm 3.6$ & $21.5 \pm 4.3$ & 0.065 & $20.9 \pm 3.8$ & $21.9 \pm 4.52$ & 0.012 \\
\hline SNAQ, points ${ }^{3}$ & $12(11-13)$ & $15(15-16)$ & $<0.001$ & $14(13-15)$ & $16(16-17)$ & $<0.001$ \\
\hline SNAQ-JE, points ${ }^{3}$ & $11(10-12)$ & $15(14-15)$ & $<0.001$ & $13(11-14)$ & $15(15-16)$ & $<0.001$ \\
\hline
\end{tabular}

\section{${ }^{1} t$-test. ${ }^{2} \chi^{2}$ test. ${ }^{3}$ Mann-Whitney U test. Abbreviations: SNAQ: Simplified Nutritional Appetite Questionnaire; SNAQ-JE: Simplified} Nutritional Appetite Questionnaire-Japanese elderly.

The prevalence of GLIM-defined malnutrition and sarcopenia among participants was $56.8 \%$ and $59.2 \%$, respectively. In the SNAQ-JE, both men and women with poor appetite had lower muscle mass ( $p=0.021$ and $p=0.002$, respectively) and lower grip strength ( $p=0.007$ and $p=0.001$, respectively) than their counterparts. Furthermore, patients who showed poor appetite in the SNAQ-JE also had lower MMSE and FIM scores ( $p=0.003$ and $p=0.001$, respectively). The prevalence of GLIM-defined malnutrition and sarcopenia was higher among patients with poor appetite than the intact group, as assessed with both tools (Table 2).

Table 2. Patients' physical characteristics according to appetite status.

\begin{tabular}{|c|c|c|c|c|c|c|}
\hline & SNAQ $<14$ & SNAQ $\geq 14$ & $p$-Value & SNAQ-JE $\leq 14$ & SNAQ-JE > 14 & $p$-Value \\
\hline \multicolumn{7}{|l|}{$\begin{array}{l}\text { Skeletal muscle mass } \\
\text { index, } \mathrm{kg} / \mathrm{m}^{21}\end{array}$} \\
\hline - Male & $6.10 \pm 1.09$ & $6.61 \pm 0.89$ & 0.009 & $6.36 \pm 0.95$ & $6.72 \pm 0.91$ & 0.021 \\
\hline - Female & $4.84 \pm 1.04$ & $5.13 \pm 1.05$ & 0.058 & $4.88 \pm 1.05$ & $5.33 \pm 1.00$ & 0.002 \\
\hline \multicolumn{7}{|l|}{ Handgrip strength, $\mathrm{kg}^{1}$} \\
\hline - Male & $24.2 \pm 7.8$ & $26.8 \pm 8.1$ & 0.106 & $24.9 \pm 7.7$ & $28.4 \pm 8.1$ & 0.007 \\
\hline - Female & $13.4 \pm 5.7$ & $15.3 \pm 5.8$ & 0.025 & $13.8 \pm 5.4$ & $16.3 \pm 6.2$ & 0.001 \\
\hline $\begin{array}{l}\text { Mini-Mental Examination } \\
\text { State, points }{ }^{2}\end{array}$ & $25(21-28)$ & $26(21-29)$ & 0.202 & $25(20-28)$ & $27(22-29)$ & 0.021 \\
\hline $\begin{array}{l}\text { Food Intake Level } \\
\text { Scale, points }{ }^{2}\end{array}$ & $8(8-9)$ & $8(8-9)$ & 0.414 & 8 (7-99) & $8(8-9)$ & 0.003 \\
\hline $\begin{array}{c}\text { Functional Independence } \\
\text { Measure, points }{ }^{2}\end{array}$ & $79(65-94)$ & $84(66-99)$ & 0.189 & 79 (61-95) & $87(72-102)$ & 0.001 \\
\hline $\begin{array}{c}\text { Malnourished } \\
(\mathrm{MNA}-\mathrm{SF} \leq 7), n(\%)^{3}\end{array}$ & $71(75.5)$ & $144(50.3)$ & $<0.001$ & $150(64.1)$ & $65(44.5)$ & $<0.001$ \\
\hline $\begin{array}{c}\text { GLIM-defined } \\
\text { malnutrition, } n(\%)^{3}\end{array}$ & $71(75.5)$ & $145(50.7)$ & $<0.001$ & $158(67.5)$ & $58(39.7)$ & $<0.001$ \\
\hline Sarcopenia, $n(\%)^{3}$ & $67(71.3)$ & $158(55.2)$ & 0.008 & $158(67.5)$ & $67(45.9)$ & $<0.001$ \\
\hline
\end{tabular}

\footnotetext{
${ }^{1}$ t-test. ${ }^{2}$ Mann-Whitney U test. ${ }^{3} \chi^{2}$ test. Abbreviations: SNAQ-JE: Simplified Nutritional Appetite Questionnaire; SNAQ: Simplified
} Nutritional Appetite Questionnaire-Japanese elderly; MNA-SF: Mini Nutritional Assessment—Short Form; GLIM: Global Leadership Initiative on Malnutrition.

Multivariate logistic regression analysis showed that poor appetite identified by the SNAQ and SNAQ-JE was an independent factor for the GLIM-defined malnutrition (odds ratio (OR) 3.004, 95\% confidence interval (CI) 1.651-5.464, $p<0.001$, OR 2.754, 
95\% CI 1.669-4.545, $p<0.001$, respectively). Only poor appetite in the SNAQ-JE was an independent predictor of sarcopenia (OR 1.940, 95\% CI 1.176-3.198, $p=0.009$ ) (Table 3).

Table 3. Results of logistic regression analysis for current GLIM-defined malnutrition and sarcopenia.

\begin{tabular}{lccc}
\hline & \multicolumn{4}{c}{ Model 1: GLIM-Defined Malnutrition } & \\
& Odds ratio & $95 \%$ confidence interval & $<$-value \\
\hline Poor appetite in SNAQ & 3.004 & $1.651-5.464$ & $<0.001$ \\
Poor appetite in SNAQ-JE & 2.754 & $1.669-4.545$ & $<0.001$ \\
\hline \multicolumn{4}{c}{ Model 2: Sarcopenia } \\
\hline Poor appetite in SNAQ & \multicolumn{2}{c}{ O5\% confidence interval } & $p$-value \\
Poor appetite in SNAQ-JE & 1.627 & $0.894-2.963$ & 0.111 \\
\hline
\end{tabular}

Model 1 adjusted for age, sex, primary disease, days from disease onset to rehabilitation start, Charlson comorbidity index, Mini Mental State Examination, Food Intake Level Scale, and Functional Independence Measure. Model 2 adjusted for age, sex, primary disease, days from disease onset to rehabilitation start, Charlson comorbidity index, Mini Nutritional Assessment Short Form $\leq 7$, Mini Mental State Examination, Food Intake Level Scale, and Functional Independence Measure. Abbreviations: SNAQ: Simplified Nutritional Appetite Questionnaire; SNAQ-JE: Simplified Nutritional Appetite Questionnaire—Japanese elderly; GLIM: Global Leadership Initiative on Malnutrition.

Table 4 shows the predictive ability of poor appetite and reference tools as predictors for GLIM-defined malnutrition and sarcopenia. Poor appetite in the SNAQ had low sensitivity (approximately 30\%) but high specificity (approximately 80\%) for GLIM-defined malnutrition and sarcopenia. Meanwhile, poor appetite in the SNAQ-JE had a sensitivity of approximately $70 \%$ and a specificity of approximately $50 \%$ for GLIM-defined malnutrition and sarcopenia. The SNAQ-JE tended to be more sensitive than the SNAQ. In addition, undernutrition, as assessed by the MNA-SF, showed a sensitivity and specificity of approximately $70 \%$ each for GLIM-defined malnutrition and sensitivity and specificity of approximately $60 \%$ each for sarcopenia.

Table 4. Accuracy of poor appetite and reference tools as predictors of current GLIM-defined malnutrition and sarcopenia.

\begin{tabular}{|c|c|c|c|c|c|c|}
\hline \multicolumn{7}{|c|}{ Poor Appetite in SNAQ } \\
\hline & Sensitivity & Specificity & Accuracy & PPV & NPV & AUC \\
\hline $\begin{array}{l}\text { GLIM-defined } \\
\text { malnutrition }\end{array}$ & 0.329 & 0.860 & 0.558 & 0.755 & 0.493 & 0.706 \\
\hline Sarcopenia & 0.298 & 0.826 & 0.513 & 0.713 & 0.513 & 0.627 \\
\hline \multicolumn{7}{|c|}{ Poor Appetite in SNAQ-JE } \\
\hline & Sensitivity & Specificity & Accuracy & PPV & NPV & AUC \\
\hline $\begin{array}{l}\text { GLIM-defined } \\
\text { malnutrition }\end{array}$ & 0.731 & 0.537 & 0.647 & 0.675 & 0.603 & 0.670 \\
\hline Sarcopenia & 0.702 & 0.510 & 0.624 & 0.675 & 0.541 & 0.616 \\
\hline \multicolumn{7}{|c|}{ Undernutrition in MNA-SF $\leq 7$} \\
\hline & Sensitivity & Specificity & Accuracy & PPV & NPV & AUC \\
\hline $\begin{array}{l}\text { GLIM-defined } \\
\text { malnutrition }\end{array}$ & 0.778 & 0.713 & 0.750 & 0.781 & 0.709 & 0.815 \\
\hline Sarcopenia & 0.680 & 0.600 & 0.647 & 0.712 & 0.564 & 0.708 \\
\hline
\end{tabular}

Abbreviations: SNAQ: Simplified Nutritional Appetite Questionnaire; PPV: positive predictive value; NPV: negative predictive value; AUC: area under curve; GLIM: Global Leadership Initiative on Malnutrition; SNAQ-JE: Simplified Nutritional Appetite Questionnaire-Japanese elderly; MNA-SF: Mini Nutritional Assessment-Short Form.

The AUC associated with the SNAQ for GLIM-defined malnutrition was greater than that associated with the SNAQ-JE (0.706 vs. 0.627, $p<0.001$ for DeLong test) (Figure 1 ). However, the AUC values of the SNAQ and SNAQ-JE for sarcopenia were comparable ( 0.670 vs. $0.616, p=0.364$ for DeLong test). Receiver operating characteristic curve analysis 
of the SNAQ and SNAQ-JE predictive value for GLIM-defined malnutrition and sarcopenia was performed. The AUC was 0.706 for the SNAQ and 0.627 for the SNAQ-JE for GLIMdefined malnutrition, and 0.670 for the SNAQ and 0.616 for the SNAQ-JE for sarcopenia. The AUC for GLIM-defined malnutrition was significantly higher in the SNAQ than in the SNAQ-JE (DeLong test $p<0.001$ ). The AUC values of the SNAQ and SNAQ-JE for sarcopenia were comparable (DeLong test $p=0.364$ ).
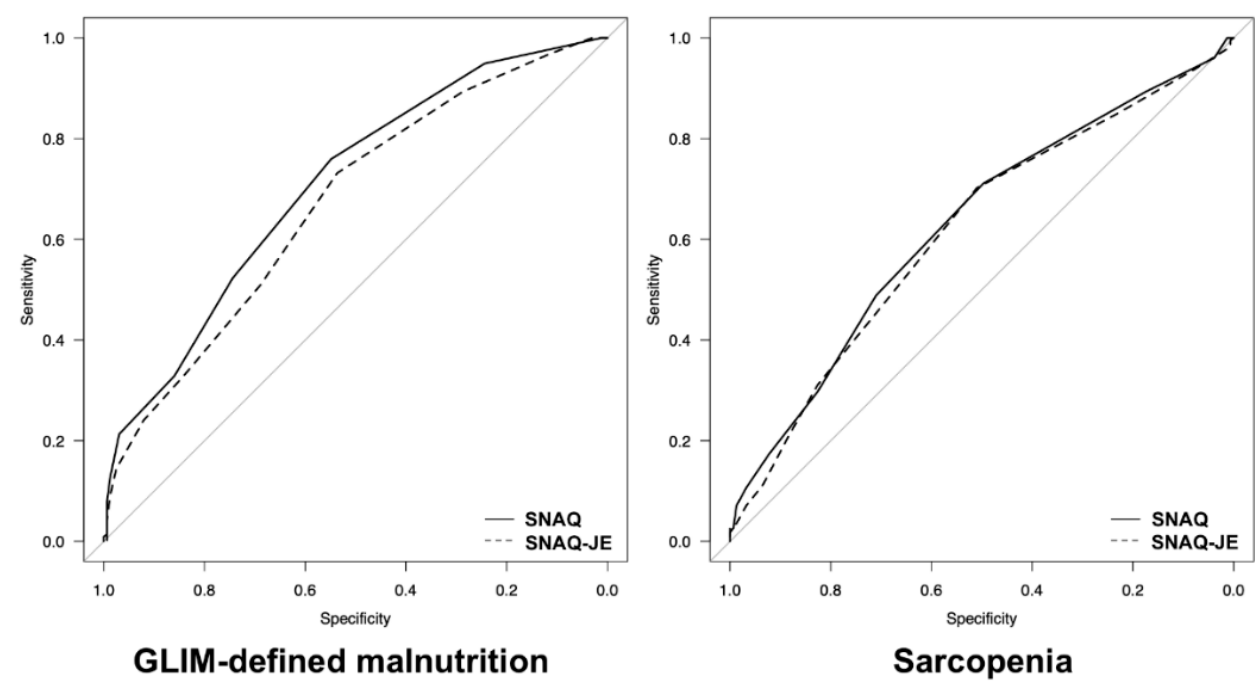

Figure 1. Comparison of the receiver-operating characteristic curves of the SNAQ and SNAQ-JE for GLIM-defined malnutrition and sarcopenia. Abbreviations: SNAQ: Simplified Nutritional Appetite Questionnaire; SNAQ-JE: Simplified Nutritional Appetite Questionnaire-Japanese elderly; GLIM: Global Leadership Initiative on Malnutrition.

\section{Discussion}

This study investigated the screening accuracy of the SNAQ and SNAQ-JE for GLIMdefined malnutrition and sarcopenia in older patients who were admitted to our rehabilitation units. The results revealed fair accuracy of the SNAQ-JE in screening for GLIM-defined malnutrition and sarcopenia.

The SNAQ-JE showed fair accuracy for detecting GLIM-defined malnutrition. In this study, the accuracy of the SNAQ for detecting GLIM-defined malnutrition was associated with sensitivity and specificity estimates of $32.9 \%$ of $86 \%$, respectively; meanwhile, the SNAQ-JE was associated with sensitivity and specificity estimates of $73.1 \%$ and $53.7 \%$, respectively. The SNAQ is a validated tool for screening undernutrition in various settings $[14,15,31]$. The accuracy of SNAQ-based screening for undernutrition assessed by the MNA in inpatients has been shown to be poor to fair, with sensitivity and specificity estimates of $28-70.8 \%$ and $74.4-94 \%$, respectively [14,15]. In contrast, the accuracy of nutritional screening tools for detecting GLIM-defined malnutrition in hospitalized patients has been shown to be fair to good, with sensitivity and specificity values of $47.1-84.1 \%$ and $47.1-93.7 \%$, respectively $[20,33,34]$. These results suggest that the accuracy of nutritional screening tools for detecting undernutrition, as defined by the GLIM, is often better than fair. In addition, given the importance of early interventions in undernutrition [35], screening tools that show high sensitivity are desirable. In the DeLong test, the SNAQ had a greater AUC than the SNAQ-JE. However, the sensitivity of the SNAQ was lower than that of the SNAQ-JE. In addition, the MNA-SF, which was used as a reference tool in this study, showed better accuracy than the SNAQ and SNAQ-JE for undernutrition as defined by the GLIM, but the screening accuracy was fair. Therefore, the SNAQ-JE, which was rated with fair accuracy, as well as the MNA-SF may be useful in screening for GLIM-defined malnutrition in older Japanese inpatients.

The SNAQ-JE showed fair accuracy for detecting sarcopenia. In this study, the accuracy of the SNAQ for detecting sarcopenia showed a sensitivity of $29.8 \%$ and specificity 
of $82.6 \%$. In comparison, the SNAQ-JE showed a sensitivity of $70.1 \%$ and specificity of $51.0 \%$. The diagnostic criteria for sarcopenia emphasize the need for screening, including SARC-F and calf circumference assessments, to enable early intervention [6,10]. A metaanalysis of studies investigating the diagnostic accuracy of the SARC-F for sarcopenia [36] in older adults reported sensitivity and specificity estimates of $21 \%(95 \%$ CI: $13-31 \%)$ and 90\% (95\% CI: 83-94\%), respectively [37]. In addition, the calf circumference assessment recommended by AWGS 2019 [10] for screening sarcopenia in older inpatients is associated with a respective sensitivity and specificity of $85 \%$ and $66 \%$ in men and $91 \%$ and $28 \%$ in women [11]. In the present study, the SNAQ for sarcopenia was associated with sensitivity and specificity values of $29.8 \%$ and $82.6 \%$, respectively; these values were similar to SARC$F$ values reported previously [37]. However, the SNAQ was not independently associated with sarcopenia in multivariate analysis. In addition, the MNA-SF, which was used as a reference tool in this study, showed better accuracy than the SNAQ and SNAQ-JE for sarcopenia, but the screening accuracy was fair. Therefore, the SNAQ-JE, which has fair accuracy for sarcopenia, may be a useful screening tool.

The SNAQ and SNAQ-JE may be useful for screening for GLIM-defined malnutrition and sarcopenia and for detecting prevention and intervention opportunities. The sensitivity and specificity values of predicting body weight loss of $\geq 5 \%$ within 6 months in patients with poor appetite identified by the SNAQ were $81.5 \%$ and $76.4 \%$, respectively [29]. In addition, previous studies have shown that the SNAQ is associated with sensitivity and specificity values for predicting body weight loss of $\geq 10 \%$ of $88.2 \%$ and $83.5 \%$, respectively [12]. Body weight loss is an essential component of malnutrition [7] and is considered a risk factor for sarcopenia [10]. The SNAQ is a simple, four-question instrument that does not require physical measurements and can help identify poor appetite in inpatients [12]. Therefore, the use of the SNAQ and SNAQ-JE in clinical practice may support early identification of poor appetite and the implementation of suitable interventions. In the current study, the distribution of "the number of meals per day" for the SNAQ was mostly concentrated in the response of "three meals per day". This result was similar to the results of a previous study that created the SNAQ-JE [13] and SNAQ Turkish version [38]. In contrast, the distribution of responses to "general mood", a component of the SNAQ-JE, was scattered. These results suggest that the low factor loading and narrow distribution of the "number of meals per day" factor of the SNAQ questionnaire shown in previous studies may be responsible for the differences in rates of poor appetite among older Japanese inpatients. Further studies are needed to investigate the predictive value of the SNAQ and SNAQ-JE in older Japanese inpatients, considering these findings.

This study has several limitations. First, the accuracy of the SNAQ and SNAQ-JE for GLIM-defined malnutrition and sarcopenia in this study was not very high. Therefore, prudence may be needed to determine the choice of nutritional therapy in individual clinical cases. Second, the study only included patients who were able to answer the SNAQ and SNAQ-JE. Therefore, the results of this study cannot be applied to all hospitalized patients. However, many comprehensive geriatric assessment tools have similar limitations [14]. Third, the accuracy of the SNAQ and SNAQ-JE may have been affected by the selective bias of the population included in this study. Approximately $60 \%$ of the participants in our study had GLIM-defined malnutrition and sarcopenia. In a previous study of Japanese patients undergoing rehabilitation, the prevalence of undernutrition and sarcopenia was approximately $40 \%$ and $50 \%$, respectively [22]. Therefore, the accuracy of the SNAQ and SNAQ-JE for these conditions should be validated in the future in a multicenter study. Finally, the SNAQ-JE used in this study was a modified version of the SNAQ validated for older Japanese adults. Therefore, it is unclear whether similar results would be obtained with the SNAQ-JE for non-Japanese populations. However, the SNAQ has different cut-off values for different regions and settings [31]; therefore, it is necessary to use the cut-off value of the SNAQ that is appropriate for the target population. 


\section{Conclusions}

The SNAQ-JE showed fair accuracy in screening for GLIM-defined malnutrition and sarcopenia in older patients admitted to rehabilitation units. Further studies are required to determine whether the SNAQ-JE can predict the development of GLIM-defined malnutrition and sarcopenia during hospitalization in older inpatients.

Author Contributions: Conceptualization, A.S., I.F., K.M. (Keisuke Maeda), K.M. (Kenta Murotani), T.I., T.O., A.N. (Ayano Nagano), J.U., Y.I., A.N. (Akiko Nomoto), J.K., and N.M.; methodology, A.S., I.F., K.M. (Keisuke Maeda), K.M. (Kenta Murotani), T.I., T.O., A.N. (Ayano Nagano), J.U., Y.I., A.N. (Akiko Nomoto), J.K., and N.M.; investigation, A.S., I.F., T.O., and A.N. (Akiko Nomoto); formal analysis, A.S., K.M. (Keisuke Maeda), and K.M. (Kenta Murotani); writing-original draft preparation, A.S., I.F., and K.M. (Keisuke Maeda); writing-review and editing, A.S., I.F., K.M. (Keisuke Maeda), K.M. (Kenta Murotani), T.I., T.O., A.N. (Ayano Nagano), J.U., Y.I., A.N. (Akiko Nomoto), J.K., and N.M. All authors have read and agreed to the published version of the manuscript.

Funding: This research was supported by the Research Funding of Longevity Sciences (Grant number: 20-57) from the Ministry of Health, Labour and Welfare of Japan K.M. (Keisuke Maeda) and the Nakatani Suzuyo Memorial Fund for Nutrition and Dietetics (A.S.).

Institutional Review Board Statement: This study was approved by the Ethics Committee of the Hamamatsu City Rehabilitation Hospital (ID: 20-63).

Informed Consent Statement: Informed consent was not obtained because of the retrospective study design. Patients could withdraw from the study at any time using an opt-out procedure.

Data Availability Statement: Data sharing is not applicable to this article.

Conflicts of Interest: The authors declare no conflict of interest.

\section{References}

1. Morley, J.E. Anorexia of aging: A true geriatric syndrome. J. Nutr. Health Aging 2012, 16, 422-425. [CrossRef]

2. Wysokiński, A.; Sobów, T.; Kłoszewska, I.; Kostka, T. Mechanisms of the anorexia of aging-A review. Age 2015, $37,9821$. [CrossRef] [PubMed]

3. Tsutsumimoto, K.; Doi, T.; Makizako, H.; Hotta, R.; Nakakubo, S.; Makino, K.; Suzuki, T.; Shimada, H. Aging-related anorexia and its association with disability and frailty. J. Cachexia Sarcopenia Muscle 2018, 9, 834-843. [CrossRef] [PubMed]

4. Morley, J.E. Anorexia of ageing: A key component in the pathogenesis of both sarcopenia and cachexia. J. Cachexia Sarcopenia Muscle 2017, 8, 523-526. [CrossRef]

5. Cox, N.J.; Ibrahim, K.; Sayer, A.A.; Robinson, S.M.; Roberts, H.C. Assessment and Treatment of the Anorexia of Aging: A Systematic Review. Nutrients 2019, 11, 144. [CrossRef]

6. Cruz-Jentoft, A.J.; Bahat, G.; Bauer, J.; Boirie, Y.; Bruyère, O.; Cederholm, T.; Cooper, C.; Landi, F.; Rolland, Y.; Sayer, A.A.; et al. Sarcopenia: Revised European consensus on definition and diagnosis. Age Ageing 2019, 48, 16-31. [CrossRef]

7. Jensen, G.L.; Cederholm, T.; Correia, M.I.T.D.; Gonzalez, M.C.; Fukushima, R.; Higashiguchi, T.; de Baptista, G.A.; Barazzoni, R.; Blaauw, R.; Coats, A.J.S.; et al. GLIM Criteria for the Diagnosis of Malnutrition: A Consensus Report From the Global Clinical Nutrition Community. JPEN J. Parenter. Enteral Nutr. 2019, 43, 32-40. [CrossRef]

8. Kaiser, M.J.; Bauer, J.M.; Ramsch, C.; Uter, W.; Guigoz, Y.; Cederholm, T.; Thomas, D.R.; Anthony, P.; Charlton, K.E.; Maggio, M.; et al. Validation of the Mini Nutritional Assessment short-form (MNA-SF): A practical tool for identification of nutritional status. J. Nutr. Health Aging 2009, 13, 782-788. [CrossRef]

9. Ye, X.-J.; Ji, Y.-B.; Ma, B.-W.; Huang, D.-D.; Chen, W.-Z.; Pan, Z.-Y.; Shen, X.; Zhuang, C.-L.; Yu, Z. Comparison of three common nutritional screening tools with the new European Society for Clinical Nutrition and Metabolism (ESPEN) criteria for malnutrition among patients with geriatric gastrointestinal cancer: A prospective study in China. BMJ Open 2018, 8, e019750. [CrossRef] [PubMed]

10. Chen, L.-K.; Woo, J.; Assantachai, P.; Auyeung, T.-W.; Chou, M.-Y.; Iijima, K.; Jang, H.C.; Kang, L.; Kim, M.; Kim, S.; et al. Asian Working Group for Sarcopenia: 2019 Consensus Update on Sarcopenia Diagnosis and Treatment. J. Am. Med. Dir. Assoc. 2020, 21, 300-307.e2. [CrossRef]

11. Inoue, T.; Maeda, K.; Shimizu, A.; Nagano, A.; Ueshima, J.; Sato, K.; Murotani, K. Calf circumference value for sarcopenia screening among older adults with stroke. Arch. Gerontol. Geriatr. 2021, 93, 104290. [CrossRef]

12. Wilson, M.-M.G.; Thomas, D.R.; Rubenstein, L.Z.; Chibnall, J.T.; Anderson, S.; Baxi, A.; Diebold, M.R.; Morley, J.E. Appetite assessment: Simple appetite questionnaire predicts weight loss in community-dwelling adults and nursing home residents. Am. J. Clin. Nutr. 2005, 82, 1074-1081. [CrossRef] 
13. Tokudome, Y.; Okumura, K.; Kumagai, Y.; Hirano, H.; Kim, H.; Morishita, S.; Watanabe, Y. Development of the Japanese version of the Council on Nutrition Appetite Questionnaire and its simplified versions, and evaluation of their reliability, validity, and reproducibility. J. Epidemiol. 2017, 27, 524-530. [CrossRef] [PubMed]

14. Rolland, Y.; Perrin, A.; Gardette, V.; Filhol, N.; Vellas, B. Screening older people at risk of malnutrition or malnourished using the Simplified Nutritional Appetite Questionnaire (SNAQ): A comparison with the Mini-Nutritional Assessment (MNA) tool. J. Am. Med. Dir. Assoc. 2012, 13, 31-34. [CrossRef]

15. Yaxley, A.; Crotty, M.; Miller, M. Identifying Malnutrition in an Elderly Ambulatory Rehabilitation Population: Agreement between Mini Nutritional Assessment and Validated Screening Tools. Healthcare 2015, 3, 822-829. [CrossRef] [PubMed]

16. Wang, T.; Shen, J. Usefulness of Simplified Nutritional Appetite Questionnaire (SNAQ) in Appetite Assessment in Elder Patients with Liver Cirrhosis. J. Nutr. Health Aging 2018, 22, 911-915. [CrossRef]

17. Wleklik, M.; Lisiak, M.; Andreae, C.; Uchmanowicz, I. Psychometric Evaluation of Appetite Questionnaires in Elderly Polish Patients with Heart Failure. Patient Prefer. Adherence 2019, 13, 1751-1759. [CrossRef] [PubMed]

18. Shimizu, A.; Maeda, K.; Honda, T.; Ishida, Y.; Ueshima, J.; Nagami, S.; Nagano, A.; Inoue, T.; Murotani, K.; Kayashita, J.; et al. Comparison between the Global Leadership Initiative on Malnutrition and the European Society for Clinical Nutrition and Metabolism definitions for the prevalence of malnutrition in geriatric rehabilitation care. Geriatr. Gerontol. Int. 2020, 20, 1221-1227. [CrossRef] [PubMed]

19. Shimizu, A.; Maeda, K.; Koyanagi, Y.; Kayashita, J.; Fujishima, I.; Mori, N. The Global Leadership Initiative on MalnutritionDefined Malnutrition Predicts Prognosis in Persons with Stroke-Related Dysphagia. J. Am. Med. Dir. Assoc. 2019, 20, 1628-1633. [CrossRef] [PubMed]

20. Clark, A.B.; Reijnierse, E.M.; Lim, W.K.; Maier, A.B. Prevalence of malnutrition comparing the GLIM criteria, ESPEN definition and MST malnutrition risk in geriatric rehabilitation patients: RESORT. Clin. Nutr. 2020, 39, 3504-3511. [CrossRef]

21. Shimizu, A.; Maeda, K.; Wakabayashi, H.; Nishioka, S.; Nagano, A.; Kayashita, J.; Fujishima, I.; Momosaki, R. Predictive Validity of Body Mass Index Cutoff Values Used in the Global Leadership Initiative on Malnutrition Criteria for Discriminating Severe and Moderate Malnutrition Based on In-Patients With Pneumonia in Asians. JPEN J. Parenter. Enteral Nutr. 2020. [CrossRef]

22. Yoshimura, Y.; Wakabayashi, H.; Bise, T.; Nagano, F.; Shimazu, S.; Shiraishi, A.; Yamaga, M.; Koga, H. Sarcopenia is associated with worse recovery of physical function and dysphagia and a lower rate of home discharge in Japanese hospitalized adults undergoing convalescent rehabilitation. Nutrition 2019, 61, 111-118. [CrossRef] [PubMed]

23. Tsutsumimoto, K.; Doi, T.; Nakakubo, S.; Kim, M.; Kurita, S.; Ishii, H.; Shimada, H. Association between anorexia of ageing and sarcopenia among Japanese older adults. J. Cachexia Sarcopenia Muscle 2020, 11, 1250-1257. [CrossRef] [PubMed]

24. Charlson, M.E.; Pompei, P.; Ales, K.L.; MacKenzie, C.R. A new method of classifying prognostic comorbidity in longitudinal studies: Development and validation. J. Chronic Dis. 1987, 40, 373-383. [CrossRef]

25. Kunieda, K.; Ohno, T.; Fujishima, I.; Hojo, K.; Morita, T. Reliability and validity of a tool to measure the severity of dysphagia: The Food Intake LEVEL Scale. J. Pain Symptom Manag. 2013, 46, 201-206. [CrossRef] [PubMed]

26. Kidd, D.; Stewart, G.; Baldry, J.; Johnson, J.; Rossiter, D.; Petruckevitch, A.; Thompson, A.J. The Functional Independence Measure: A comparative validity and reliability study. Disabil. Rehabil. 1995, 17, 10-14. [CrossRef] [PubMed]

27. Folstein, M.F.; Folstein, S.E.; McHugh, P.R. "Mini-mental state". A practical method for grading the cognitive state of patients for the clinician. J. Psychiatr. Res. 1975, 12, 189-198. [CrossRef]

28. Nakatsu, N.; Sawa, R.; Misu, S.; Ueda, Y.; Ono, R. Reliability and validity of the Japanese version of the simplified nutritional appetite questionnaire in community-dwelling older adults. Geriatr. Gerontol. Int. 2015, 15, 1264-1269. [CrossRef] [PubMed]

29. Phillips, M.B.; Foley, A.L.; Barnard, R.; Isenring, E.A.; Miller, M.D. Nutritional screening in community-dwelling older adults: A systematic literature review. Asia Pac. J. Clin. Nutr. 2010, 19, 440-449.

30. Yoshimura, Y.; Wakabayashi, H.; Nagano, F.; Bise, T.; Shimazu, S.; Shiraishi, A. Chair-stand exercise improves post-stroke dysphagia. Geriatr. Gerontol. Int. 2020, 20, 885-891. [CrossRef]

31. Lau, S.; Pek, K.; Chew, J.; Lim, J.P.; Ismail, N.H.; Ding, Y.Y.; Cesari, M.; Lim, W.S. The Simplified Nutritional Appetite Questionnaire (SNAQ) as a Screening Tool for Risk of Malnutrition: Optimal Cutoff, Factor Structure, and Validation in Healthy CommunityDwelling Older Adults. Nutrients 2020, 12, 2885. [CrossRef]

32. Power, L.; Mullally, D.; Gibney, E.R.; Clarke, M.; Visser, M.; Volkert, D.; Bardon, L.; de van der Schueren, M.A.E.; Corish, C.A. MaNuEL Consortium A review of the validity of malnutrition screening tools used in older adults in community and healthcare settings-A MaNuEL study. Clin. Nutr. ESPEN 2018, 24, 1-13. [CrossRef]

33. Bellanti, F.; Lo Buglio, A.; Quiete, S.; Pellegrino, G.; Dobrakowski, M.; Kasperczyk, A.; Kasperczyk, S.; Vendemiale, G. Comparison of Three Nutritional Screening Tools with the New Glim Criteria for Malnutrition and Association with Sarcopenia in Hospitalized Older Patients. J. Clin. Med. Res. 2020, 9, 1898. [CrossRef]

34. Balci, C.; Bolayir, B.; Eşme, M.; Arik, G.; Kuyumcu, M.E.; Yeşil, Y.; Varan, H.D.; Kara, Ö.; Güngör, A.E.; Doğu, B.B.; et al Comparison of the Efficacy of the Global Leadership Initiative on Malnutrition Criteria, Subjective Global Assessment, and Nutrition Risk Screening 2002 in Diagnosing Malnutrition and Predicting 5-Year Mortality in Patients Hospitalized for Acute Illnesses. JPEN J. Parenter. Enteral Nutr. 2020. [CrossRef]

35. Schuetz, P.; Fehr, R.; Baechli, V.; Geiser, M.; Deiss, M.; Gomes, F.; Kutz, A.; Tribolet, P.; Bregenzer, T.; Braun, N.; et al. Individualised nutritional support in medical inpatients at nutritional risk: A randomised clinical trial. Lancet 2019, 393, 2312-2321. [CrossRef] 
36. Malmstrom, T.K.; Morley, J.E. SARC-F: A simple questionnaire to rapidly diagnose sarcopenia. J. Am. Med. Dir. Assoc. 2013, 14, 531-532. [CrossRef]

37. Ida, S.; Kaneko, R.; Murata, K. SARC-F for Screening of Sarcopenia among Older Adults: A Meta-analysis of Screening Test Accuracy. J. Am. Med. Dir. Assoc. 2018, 19, 685-689. [CrossRef] [PubMed]

38. İlhan, B.; Bahat, G.; Oren, M.M.; BKılıç, C.; Durmazoglu, S.; Karan, M.A. Reliability and validity of Turkish version of the Simplified Nutritional Appetite Questionnaire (SNAQ). J. Nutr. Health Aging 2018, 22, 1039-1044. [CrossRef] [PubMed] 